\title{
Phase-Shift Keying Modulated Data Signal Using SOA-MZI-Based All-Optical Logic AND Gate at $80 \mathrm{~Gb} / \mathrm{s}$
}

\author{
Amer Kotb $\mathbb{D i D}^{1,2}$ and Yasser Mohamed ${ }^{2}$ \\ ${ }^{1}$ The Guo China-US Photonics Laboratory, Changchun Institute of Optics, Fine Mechanics, and Physics, \\ Chinese Academy of Sciences, Changchun 130033, China \\ ${ }^{2}$ Department of Physics, Faculty of Science, University of Fayoum, Fayoum 63514, Egypt
}

Correspondence should be addressed to Amer Kotb; amer_22003@yahoo.com

Received 24 January 2018; Accepted 12 February 2018; Published 9 July 2018

Academic Editor: Gong-Ru Lin

Copyright (C) 2018 Amer Kotb and Yasser Mohamed. This is an open access article distributed under the Creative Commons Attribution License, which permits unrestricted use, distribution, and reproduction in any medium, provided the original work is properly cited.

\begin{abstract}
All-optical logic AND operation with phase-shift keying (PSK) modulated data signals based on a semiconductor optical amplifier (SOA) assisted Mach-Zehnder interferometer is numerically analyzed and investigated. By solving the rate equations of the SOA, the dependence of the quality factor on the critical parameters of the input signal and the SOA is examined, including the impact of the amplified spontaneous emission to obtain realistic results. The obtained results confirm that the all-optical logic AND scheme with PSK modulated data signals is capable of operating at a data rate of $80 \mathrm{~Gb} / \mathrm{s}$ with both logical correctness and acceptable quality compared to the system using on-off-keying signal.
\end{abstract}

\section{Introduction}

Modulation is the transfer process of information over a medium. Recently, advanced optical modulation formats have attracted increased attention. Some of these formats carry information through the amplitude, which is known as amplitude modulation (AM). In AM, the amplitude of the carrier changes in response to the information by keeping the frequency and the phase constants. In many applications, AM is being replaced by frequency and phase modulation technologies even though it is not as widely used as it was in previous years. However, AM is not efficient in terms of its use of power and bandwidth and is prone to high levels of noise. In contrast, phase modulation (PM) signals convey the information in the phase itself. PM schemes are attracting much interest for use in the ultrafast optical communications systems because they are less sensitive to nonlinear fiber transmission impairments, require lower optical signal-to-noise ratio, are more robust against pattern-dependent degradations, and can give higher spectral efficiency compared to conventional intensity modulation formats $[1,2]$. This fact has spurred great interest in conducting all-optical operations on phaseshift keying (PSK) modulated data so as to realize exclusively by the means of the light critical network functionalities that are compatible with the specific format. All-optical modulation based on nonlinear silicon photonics waveguide has been demonstrated [3-7]. Such all-optical logic operations are an integral part of building all-optical data networks, where packet routing, data buffering, and wavelength conversion are expected to be processed in the optical plane. For this purpose, a technical option that has been widely adopted is to employ semiconductor optical amplifiers (SOAs). In fact, the SOAs' devices have technologically matured to the point that they have become the primary choice for the implementation of all-optical logic gates. SOA is the most attractive optoelectronics device due to its compact size, low power requirement, temperature stability, and ease to connect with other optoelectronics devices. In recent years, the performances of the all-optical logic AND gate based on several different schemes using SOA have been reported [8-26]. The AND is involved in the accomplishment of numerous tasks in the optical domain both in fundamental and in systemoriented level [26]. Various SOA-based schemes for the demonstration of all-optical logic gates using PSK modulated data signals have been reported [27-33]. This activity has also included the execution of the Boolean AND operation on the 
PSK modulated data signals [31], which has been possible owing to the capability of the SOAs to handle the phase modulated signals as well [32]. However, these demonstrations cannot conveniently keep pace with the excessive increase in single-channel data rates due to the slow SOA's gain and phase recovery. The performances of all-optical logic XOR and OR gates for $100 \mathrm{~Gb} / \mathrm{s}$ PSK modulated data signal in SOAs assisted Mach-Zehnder interferometer (MZI) have been numerically analyzed $[34,35]$. In this work, we continue and generalize the relevant results of previous works [31, 35] by investigating the performance of an all-optical AND gate using PSK modulated data signals based on SOAs-MZI at a data rate of $80 \mathrm{~Gb} / \mathrm{s}$. The dependence of the gate's quality factor $(\mathrm{QF})$ on the critical parameters of the input signals and the SOAs has been examined and assessed. For realistic results, the effect of the amplified spontaneous emission (ASE) on the QF has also been included. The outcome of our work complements the set of all-optical gates that have been addressed so far for PSK signals.

\section{SOA-MZI Model}

The schematic diagram and truth table for AND operation is shown in Figure 1, where an optical band-pass filter (OBPF) is used to reject out the spectral components other than the switched signal.

The semiconductor materials used in the SOA should be direct band-gap and match lattices for the radiative recombination processes. The semiconductor materials used in this simulation are InGaAsP/InP with wavelengths of $1300 \mathrm{~nm}$ or $1550 \mathrm{~nm}$. The operation of the SOA-based MZI can be theoretically studied by means of the rate equation model [8-12]. This simulation has been prepared and run via Wolfram Mathematica. This simulation takes into account the nonlinear effects of the interband and intraband. More specifically, the carrier heating $(\mathrm{CH})$ results from thermalization of carriers in the entire energy-band following the pulse. This is a fast process occurring in the time scale of $300 \mathrm{fs}$. The input pulse reduces the gain at the photon-energy of this pulse; that is, in the gain spectrum it burns a hole. The process is known as a spectral hole burning (SHB). By taking into consideration both the $\mathrm{CH}$ and $\mathrm{SHB}$ effects, the time-dependent gain for each SOA is given by $[9,23]$

$$
\begin{aligned}
& \frac{d h_{\mathrm{CD}}(t)}{d t}=\frac{h_{0}-h_{\mathrm{CD}}(t)}{\tau_{\mathrm{C}}} \\
& -\left(\exp \left[h_{\mathrm{CD}}(t)+h_{\mathrm{CH}}(t)+h_{\mathrm{SHB}}(t)\right]-1\right) \frac{P(t)}{E_{\mathrm{sat}}}, \\
& \frac{d h_{\mathrm{CH}}(t)}{d t}=-\frac{h_{\mathrm{CH}}(t)}{\tau_{\mathrm{CH}}} \\
& -\frac{\varepsilon_{\mathrm{CH}}}{\tau_{\mathrm{CH}}}\left(\exp \left[h_{\mathrm{CD}}(t)+h_{\mathrm{CH}}(t)+h_{\mathrm{SHB}}(t)\right]-1\right) P(t), \\
& \frac{d h_{\mathrm{SHB}}(t)}{d t}=-\frac{h_{\mathrm{SHB}}(t)}{\tau_{\mathrm{SHB}}}
\end{aligned}
$$

$$
\begin{aligned}
& -\frac{\varepsilon_{\mathrm{SHB}}}{\tau_{\mathrm{SHB}}}\left(\exp \left[h_{\mathrm{CD}}(t)+h_{\mathrm{CH}}(t)+h_{\mathrm{SHB}}(t)\right]-1\right) P(t) \\
& -\frac{d h_{\mathrm{CD}}(t)}{d t}-\frac{d h_{\mathrm{CH}}(t)}{d t} .
\end{aligned}
$$

The total gain $G(t)$ of each SOA is given by

$$
G(t)=\exp \left[h_{\mathrm{CD}}(t)+h_{\mathrm{CH}}(t)+h_{\mathrm{SHB}}(t)\right],
$$

where function " $h$ " is the SOA's gain integrated over its length due to the carrier depletion (CD), CH, and SHB. $G_{0}=$ $\exp \left[h_{0}\right]$ is the unsaturated power gain. $E_{\text {sat }}$ is the saturation energy, which is linked to the saturation power $\left(P_{\text {sat }}\right)$ through $E_{\text {sat }}=P_{\text {sat }} \tau_{c}$, where $\tau_{c}$ is the carrier lifetime. $P(t)$ is input optical power injected into the SOAs. $\tau_{\mathrm{CH}}$ and $\tau_{\mathrm{SHB}}$ are the temperature relaxation rate and the carrier-carrier scattering rate, respectively. $\varepsilon_{\mathrm{CH}}$ and $\varepsilon_{\mathrm{SHB}}$ are the nonlinear gain suppression factors due to $\mathrm{CH}$ and $\mathrm{SHB}$, respectively.

The carrier density-induced phase change of each SOA is given by [23]

$$
\Phi(t)=-0.5\left[\alpha h_{\mathrm{CD}}(t)+\alpha_{\mathrm{CH}} h_{\mathrm{CH}}(t)\right]
$$

where $\alpha$ is the traditional linewidth enhancement factor, $\alpha_{\mathrm{CH}}$ is the linewidth enhancement due to $\mathrm{CH}$, and $\alpha_{\mathrm{SHB}}$ is the linewidth enhancement due to SHB. The contribution $\alpha_{\mathrm{SHB}}$ is almost null since SHB produces a nearly symmetrical spectral hole centered at the signal wavelength. Kramers-Kronig integrand becomes antisymmetric around the operating frequency and its integral remains small [24].

The input pulses used in this simulation are assumed to be Gaussian-shaped pulses, which have power described by [9]

$$
\begin{aligned}
& P_{\mathrm{A}, \mathrm{B}}(t) \equiv P_{i n}(t) \\
& =\sum_{n=-\infty}^{n=+\infty} a_{n \mathrm{~A}, \mathrm{~B}} \frac{2 \sqrt{\ln (2)} E_{0}}{\sqrt{\pi} \tau_{\mathrm{FWHM}}} \exp \left(-\frac{4 \ln (2)(t-n T)^{2}}{\tau_{\mathrm{FWHM}}^{2}}\right),
\end{aligned}
$$

where $a_{n \mathrm{~A}, \mathrm{~B}}$ stands for the $n$th pulse, which can take the logical value of " 1 " or " 0 " with equal probability. $E_{0}$ is the input pulse energy and $\tau_{\text {FWHM }}$ is the pulse width (full-width at halfmaximum). $n$ is the length of the pseudorandom binary sequence (PRBS), 127 bit, and $T$ is the bit period.

The modulated PSK signals injected into the SOAs have the following power function [36]:

$$
P_{\mathrm{PSK}}(t)=\exp \left(J \pi P_{\mathrm{A}, \mathrm{B}}(t)\right) .
$$

The split components of the PSK modulated signal B suffer the optical properties of the SOAs that have been perturbed by the respective data in the two arms of the MZI. When they interfere, the resultant AND output obeys [23]

$$
\begin{aligned}
& P_{\mathrm{AND}}(t)=0.25 P_{\mathrm{PSKB}}(t)\left(G_{1}(t)+G_{2}(t)\right. \\
& \left.-2 \sqrt{G_{1}(t) G_{2}(t)} \cos \left[\Phi_{1}(t)-\Phi_{2}(t)\right]\right),
\end{aligned}
$$




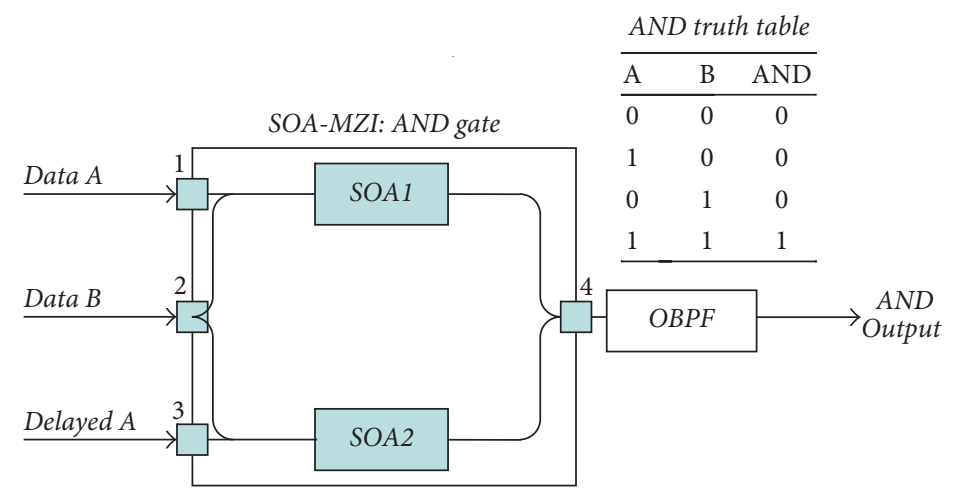

FIgURE 1: Schematic diagram and truth table for AND operation based on SOA-MZI.

where $P_{\mathrm{PSKB}}$ is the modulated PSK input power of signal $\mathrm{B}$. $G_{1,2}(t)$ and $\Phi_{1,2}(t)$ are the time-dependent total gains and the phase changes of the signal B inside SOA1 and SOA2, respectively.

The parameters used in this simulation are $I=100 \mathrm{~mA}$, $G_{0}=30 \mathrm{~dB}, P_{0}=0.006 \mathrm{pJ}, \tau_{\mathrm{FWHM}}=1.5 \mathrm{ps}, \tau_{c}=200 \mathrm{ps}, \tau_{\mathrm{CH}}=$ $0.3 \mathrm{ps}, \tau_{\mathrm{SHB}}=0.1 \mathrm{ps}, \varepsilon_{\mathrm{CH}}=\varepsilon_{\mathrm{SHB}}=0.08 \mathrm{~W}^{-1}, \alpha=4, \alpha_{\mathrm{CH}}=1$, and $P_{\text {sat }}=25 \mathrm{~mW}$. The instantaneous optical power inside each SOA for the AND operation is given by

$$
\begin{aligned}
& P_{1}(t)=P_{\mathrm{PSKA}}(t)+0.5 P_{\mathrm{PSKB}}(t), \\
& P_{2}(t)=P_{\text {Delayed PSKA }}(t)+0.5 P_{\mathrm{PSKB}}(t) .
\end{aligned}
$$

At zero injected current, the spontaneous emission process appeared and is amplified through the optically active region of the amplifier. The amplifiers degrade the SNR of the amplified signal primarily because of ASE, which is added to the signal in this simulation. The ASE can be used to measure the position of the gain peak, the pulse width of the gain curve, and the gain ripple. The power of the ASE has been numerically added to the output power of the AND operation using the following basic equation $[23,37]$ :

$$
P_{\mathrm{ASE}}=N_{\mathrm{SP}}\left(G_{0}-1\right) K v B_{0},
$$

where $K$ is Planck's constant, $B_{0}$ is the optical bandwidth, and $v$ is the optical frequency.

\section{All-Optical AND Model}

In order to realize the AND operation, we have used a similar scheme to that in $[8,10]$. The logic AND operation is achieved between the two PSK modulated data signals by means of cross-phase modulation (XPM) in SOAs 1 and 2 located in the two arms of the MZI. The input data A is injected into the upper arm of the SOA-MZI, while its delayed copy with a time delay $(\tau)$ is injected into the lower arm. Concurrently, data B at wavelength $\lambda_{2}$ acts as enabling beam and is injected into the configuration of the middle port of the MZI. This signal is halved into two components of equal amplitude and then is injected into the middle arm of the MZI. The input signals $A$ and $B$ have two different arbitrary PRBS. Initially, the two arms of the MZI are balanced in phase and the output is zero.
Then data $\mathrm{A}$ and its delayed replica are combined to create a phase window experienced by data $\mathrm{B}$. When $\mathrm{A}=$ " 0 ", this phase window does not exist, and the output is " 0 " for both B $=$ " 1 " and $\mathrm{B}=$ " 0 ". However, when $\mathrm{A}=$ " 1 ," the phase window opens allowing the split copies of signal $\mathrm{B}=$ "1" to interfere nondestructively, which results in a nonzero output. Thus, according to this switching mode, the circuit is produced " 1 " at $\lambda_{2}$ only when both data A and B are "1", which is functionally the same as an all-optical AND gate.

\section{Results}

The operation of the AND gate has been analyzed using a numerical solution of the SOA's rate equations in (1). Figure 2 illustrates the simulation results and the eye diagram for the Boolean AND operation, from which it can be noticed that the eye is clear and open. The eye diagram is with the degrading effects while the pseudo-eye diagram is without any degrading effects. The QF gives information for the optical signal-to-noise ratio (SNR) in digital transmission. The performance of the AND operation has been evaluated by the measurement of the $\mathrm{QF}$, which is given by $\mathrm{QF}=\left(P_{1}-P_{0}\right) /\left(\sigma_{1}+\right.$ $\sigma_{2}$ ), where $P_{1,0}$ are the average powers of the expected "1"s and " 0 " $s$ and $\sigma_{1,2}$ are the corresponding standard deviations. The QF gives the information of the optical SNR in digital transmission. In this simulation, the achieved QF is 34.6 at $80 \mathrm{~Gb} / \mathrm{s}$, which is well over the critical limit of 6 required to keep the bit-error-rate (BER) less than $10^{-9}$ [23]. In order to obtain realistic results, the metric characteristics of the AND operation, such as the BER and the extinction ration (ER), have been calculated. The BER is linked to the QF through the following basic equation [23]:

$$
\mathrm{BER}=0.5 \operatorname{erfc}\left[\frac{\mathrm{QF}}{\sqrt{2}}\right],
$$

where erfc is the error-function. The calculated BER in this simulation is $1.26 \times 10^{-262}$.

The ER is defined in $\mathrm{dB}$ by the following basic equation $[38,39]$ :

$$
\mathrm{ER}(\mathrm{dB})=10 \log _{10}\left[\frac{P_{1}^{\min }}{P_{0}^{\max }}\right],
$$



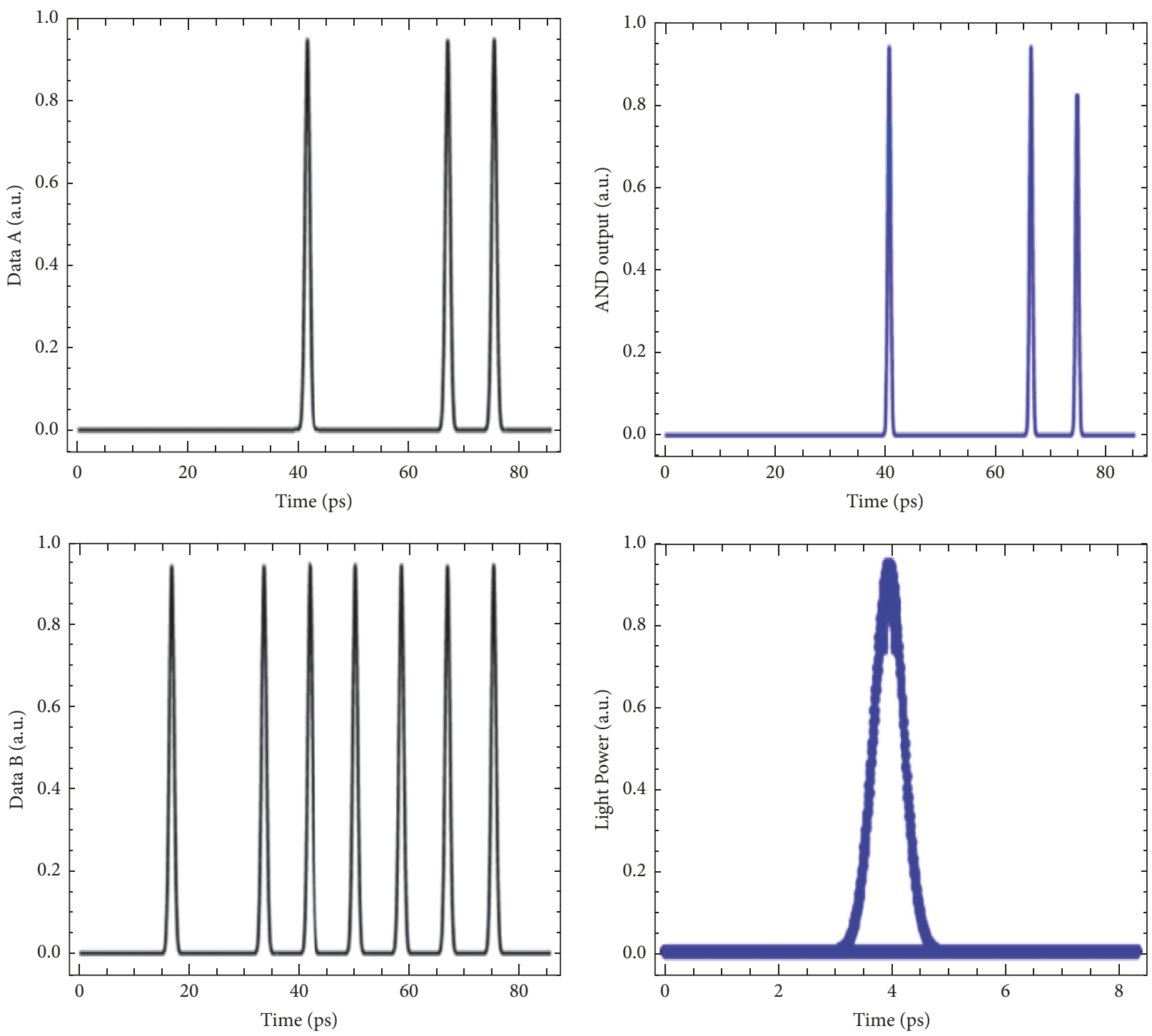

FIgURE 2: Simulation results and the corresponding eye diagram for AND operation. The achieved QF is 34.6 at a data rate of $80 \mathrm{~Gb} / \mathrm{s}$.

where $P_{1}^{\min }$ is the minimum value of the peak power of logic " 1 " and $P_{0}^{\max }$ is the maximum value of the peak power of logic " 0 ". In this simulation, the calculated ER using (10) is around $40 \mathrm{~dB}$.

The simulated QF depends on the pulse energy and the injected SOA injection current of SOA-MZI-based for the AND operation at $N_{\mathrm{sp}}=2$ and 4 is shown, respectively, in Figures 3(a) and 3(b). As shown in Figure 3(a), a high input pulse energy causes a strong saturation, which reduces the value of the QF. On the other hand, the $\mathrm{QF}$ is increased as more carriers are supplied via higher bias currents as shown in Figure 3(b). The injection current plays an important role in the amplification process. Moreover, the injection current required to obtain a $\mathrm{QF}$ of 34.6 is a few tens of $\mathrm{mAs}$ with the PSK modulated signals using SOA compared to hundreds of $\mathrm{mAs}$ with the SOA based on other modulation formats [23].

Figures 4(a) and 4(b) show the dependence of the QF on the SOA's carrier lifetime $\left(\tau_{c}\right)$ and the pulse width $\left(\tau_{\mathrm{FWHM}}\right)$ at $I=50 \mathrm{~mA}$ and $100 \mathrm{~mA}$, respectively. For a standard SOA, the value of the carrier lifetime varies from $100 \mathrm{ps}$ to $300 \mathrm{ps}$ depending on the type of the semiconductor material [23]. In our simulation case, the value of this parameter can be changed several times to calculate the corresponding QF for each value. By this way, the value of the carrier lifetime, which makes high QF, can be easily optimized. Since the carrier lifetime determines the speed of gain recovery of the SOA [23], the QF is higher for smaller values of this parameter as shown in Figure 4(a). A similar trend is observed in Figure 4(b), where the QF is decreased with increasing the pulse width. The QF is decreased with increasing the pulse width because two neighboring pulses tend to overlap for wider pulse widths.

The calculated QF depends on the length and the thickness of the SOA's active region as shown in Figures 5(a) and 5(b), respectively. The typical length and thickness of the standard SOA are $2 \mathrm{~mm}$ and $0.4 \mu \mathrm{m}$, respectively [23]. By 


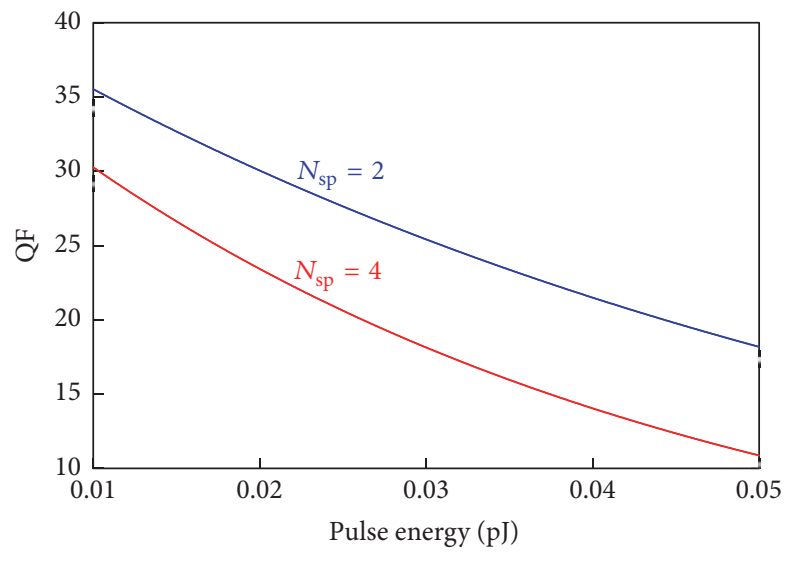

(a)

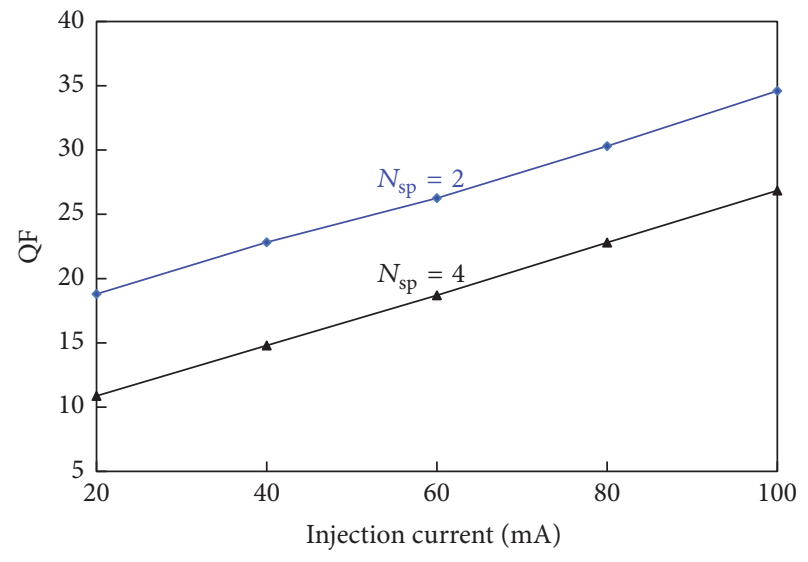

(b)

FIGURE 3: QF versus (a) pulse energy and (b) SOA's injection current for AND operation at $N_{\mathrm{sp}}=2$ and 4.

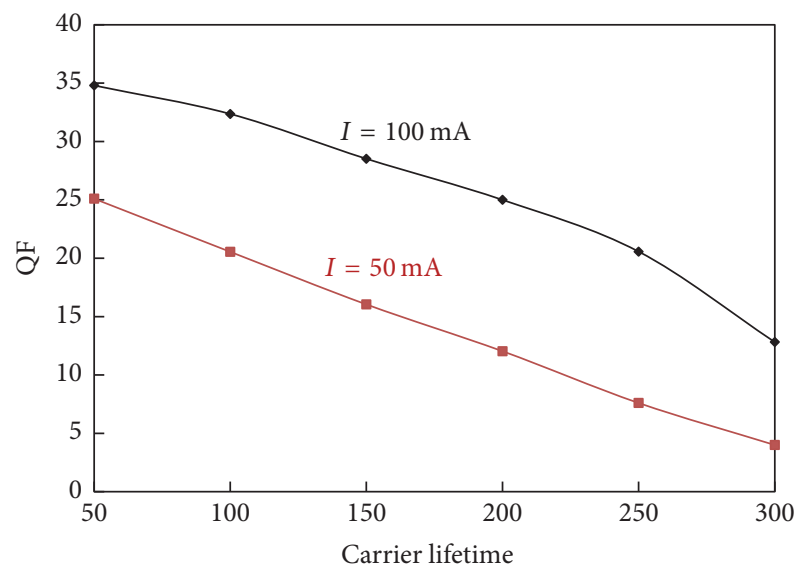

(a)

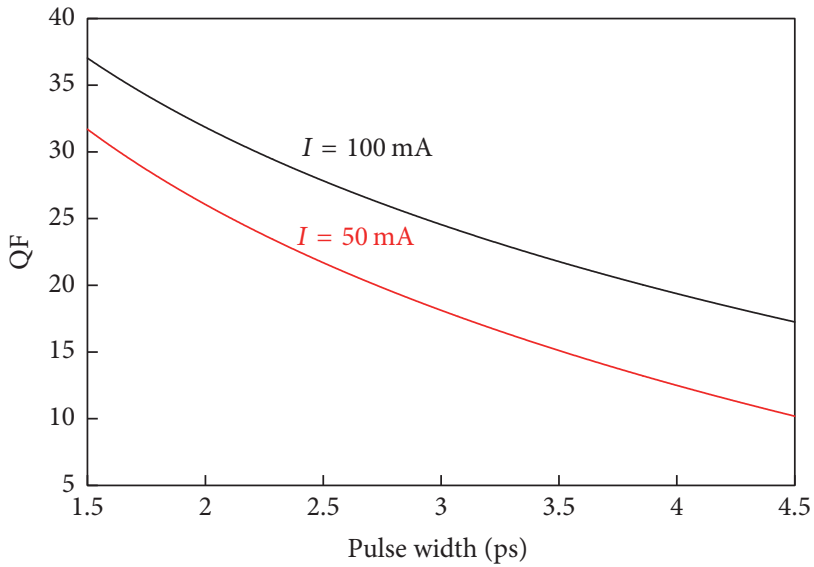

(b)

FIGURE 4: QF versus (a) SOA's carrier lifetime and (b) pulse width for AND operation.

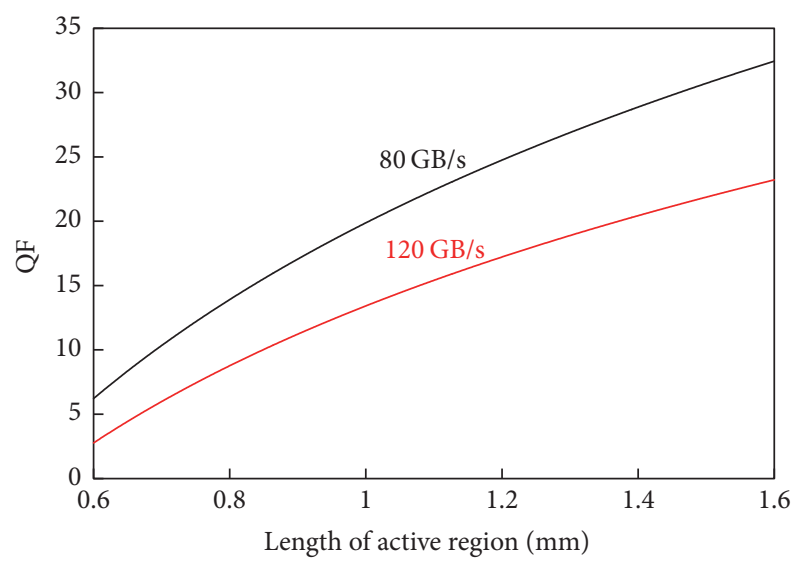

(a)

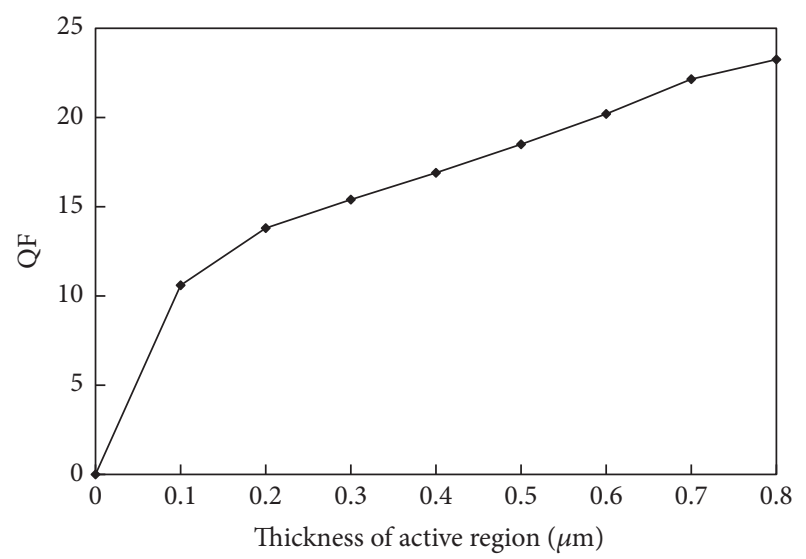

(b)

FIGURE 5: QF versus SOA's active region (a) length at $80 \mathrm{~Gb} / \mathrm{s} \& 120 \mathrm{~Gb} / \mathrm{s}$ and (b) thickness for AND operation. 


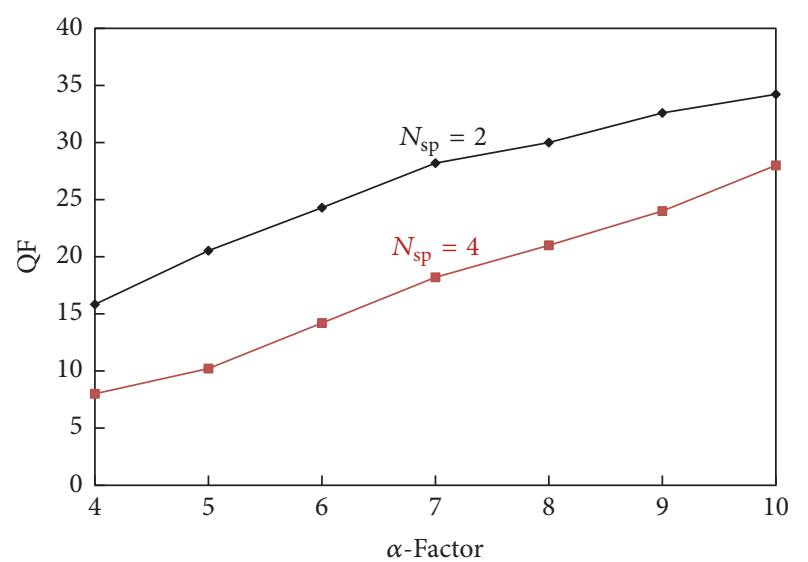

FIGURE 6: QF versus SOA's traditional linewidth enhancement factor $\left(\alpha\right.$-factor) for AND operation at $N_{\mathrm{sp}}=2$ and 4 .

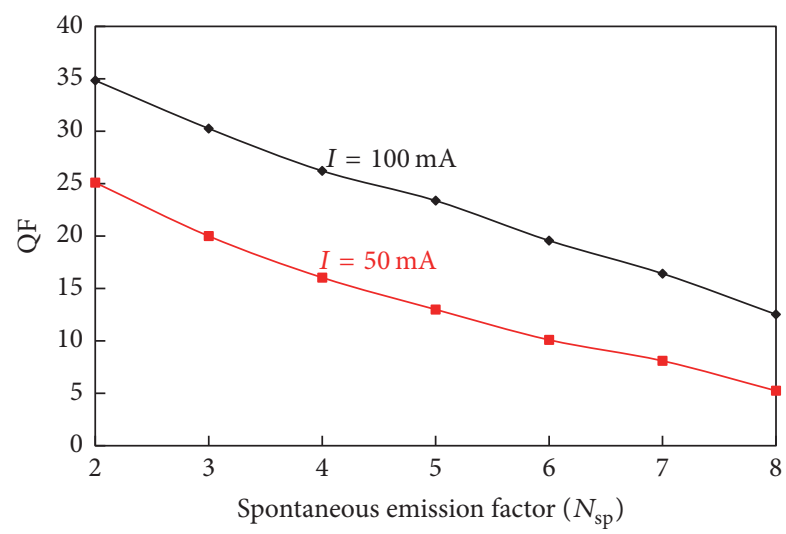

FIGURE 7: QF versus spontaneous emission factor $\left(N_{\mathrm{sp}}\right)$ of SOAMZI-based AND operation at $I=50 \mathrm{~mA}$ and $100 \mathrm{~mA}$.

increasing the length of the SOA, the overall gain increases exponentially and then the QF as shown in Figure 5(a), which has been measured at two different data rates. A thick active region is used for fast gain recovery and hence an acceptable performance of the Boolean functions. The optical gain region is determined by the active region thickness. The carrier density is increased with increasing the thickness of the active region and hence the QF as shown in Figure 5(b).

The dependence of the QF on the traditional linewidth enhancement factor ( $\alpha$-factor) at $N_{\text {sp }}=2$ and 4 is shown in Figure 6. This figure confirms that the QF is increased with $\alpha$ factor due to the effect of this parameter on the induced phase change and accordingly on the magnitude of switching [40]. Moreover, for the PSK modulated signals, the QF is higher for a small $\alpha$-factor.

The QF versus $N_{\text {sp }}$ of a SOA-MZI-based AND operation at $I=50 \mathrm{~mA}$ and $100 \mathrm{~mA}$ is shown in Figure 7. It is clearly seen that the QF is decreased with increasing the $N_{\mathrm{SP}}$, which is considered as a noise. This simulation has been carried out at $G_{0}=30 \mathrm{~dB}, B_{0}=3 \mathrm{~nm}$, and a signal wavelength of $1550 \mathrm{~nm}$. The effect of ASE is experimentally verified by adding a few nm wide optical unmodulated signals to the streams data with negligible saturation effects.

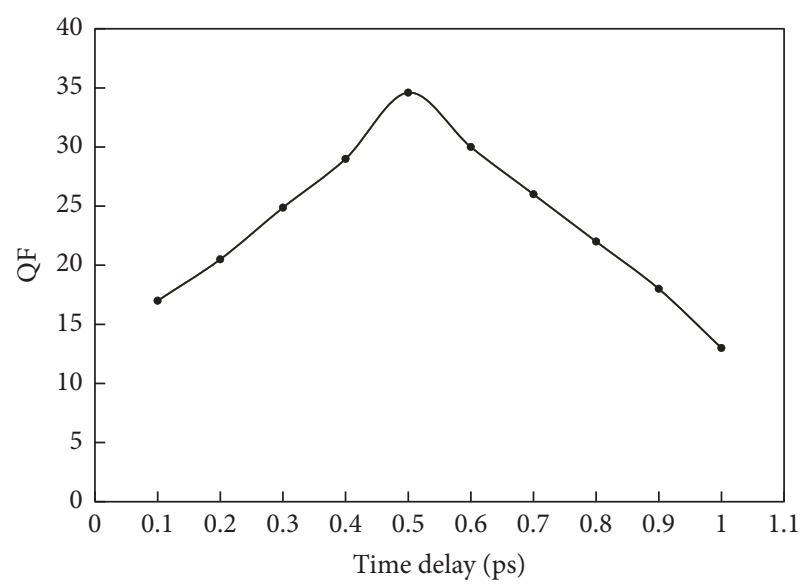

FIGURE 8: QF versus time delay for AND operation.

The time delay $(\tau)$ of delayed data A is a very important parameter for the performance of the AND gate. The optimum time delay used in this simulation that maximizes the $\mathrm{QF}$ to almost 34 is $0.5 \mathrm{ps}$. Figure 8 shows that a shorter $\tau$ leads to a sharp fall of the QF. At high $\tau$, the magnitude of the phase switching window between the data $\mathrm{A}$ and its delayed decreases and hence the corresponding $\mathrm{QF}$.

\section{Conclusion}

In conclusion, the performance of ultrafast all-optical logic AND operation with phase-shift keying (PSK) modulated data signals in semiconductor optical amplifier (SOA) assisted Mach-Zehnder interferometer was numerically simulated. The obtained results show that the all-optical logic AND gate based on PSK modulated data signals was capable of operating at a data rate of $80 \mathrm{~Gb} / \mathrm{s}$ with higher output quality factor than that used for SOA based on other modulation formats.

\section{Conflicts of Interest}

The authors declare that they have no conflicts of interest.

\section{References}

[1] K. Mishina, S. Kitagawa, and A. Maruta, "All-optical modulation format conversion from on-off-keying to multiple-level phase-shift-keying based on nonlinearity in optical fiber," Optics Express, vol. 15, no. 13, pp. 8444-8453, 2007.

[2] G. Charlet, "Progress in optical modulation formats for highbit rate WDM transmissions," IEEE Journal of Selected Topics in Quantum Electronics, vol. 12, no. 4, pp. 469-483, 2006.

[3] C.-L. Wu, S.-P. Su, and G.-R. Lin, "All-optical modulation based on silicon quantum dot doped SiOx: SI-QD waveguide," Laser \& Photonics Reviews, vol. 8, no. 5, pp. 766-776, 2014.

[4] Q. Xu and M. Lipson, "All-optical logic based on silicon microring resonators," Optics Express, vol. 15, no. 3, pp. 924-929, 2007.

[5] R. Osgood, J. B. Driscoll, W. Astar et al., "Nonlinear silicon photonics," Nature Photonics, vol. 4, pp. 535-544, 2010. 
[6] D. T. Spencer, J. F. Bauters, M. J. R. Heck, and J. E. Bowers, "Integrated waveguide coupled Si3N4 resonators in the ultrahigh-Q regime," Optica, vol. 1, no. 3, pp. 153-157, 2014.

[7] G.-R. Lin, S.-P. Su, C.-L. Wu et al., "Si-rich SiNx based Kerr switch enables optical data conversion up to $12 \mathrm{Gbit} / \mathrm{s}$," Scientific Reports, vol. 5, Article ID 9611, 2015.

[8] A. Kotb, S. Ma, Z. Chen, N. K. Dutta, and G. Said, "Effect of amplified spontaneous emission on semiconductor optical amplifier based all-optical logic," Optics Communications, vol. 284, no. 24, pp. 5798-5803, 2011.

[9] A. Kotb, All-Optical Logic Gates Using Semiconductor Optical Amplifiers, Lambert Academic Publishing, Germany, 2012.

[10] A. Kotb, "AND gate based on two-photon absorption in semiconductor optical amplifier," Optoelectronics Letters, vol. 9, no. 3, pp. 181-184, 2013.

[11] A. Kotb and K. E. Zoiros, "On the design of all-optical gates based on quantum-dot semiconductor optical amplifier with effect of amplified spontaneous emission," Optical and Quantum Electronics, vol. 46, no. 8, pp. 977-989, 2014.

[12] A. Kotb, "Simulation of high quality factor all-optical logic gates based on quantum-dot semiconductor optical amplifier at 1 $\mathrm{Tb} / \mathrm{s}$, " Optik-International Journal for Light and Electron Optics , vol. 127, no. 1, pp. 320-325, 2016.

[13] H. Dong, H. Sun, Q. Wang, N. K. Dutta, and J. Jaques, " $80 \mathrm{~Gb} / \mathrm{s}$ All-optical logic AND operation using Mach-Zehnder interferometer with differential scheme," Optics Communications, vol. 265, no. 1, pp. 79-83, 2006.

[14] H. Dong, H. Sun, Q. Wang, N. K. Dutta, and J. Jaques, “Alloptical logic and operation at $80 \mathrm{~Gb} / \mathrm{s}$ using semiconductor optical amplifier based on the Mach-Zehnder interferometer," Microwave and Optical Technology Letters, vol. 48, no. 8, pp. 1672-1675, 2006.

[15] H. Sun, Q. Wang, H. Dong, and N. K. Dutta, "All-optical logic performance of quantum-dot semiconductor amplifier-based devices," Microwave and Optical Technology Letters, vol. 48, no. 1, pp. 29-35, 2006.

[16] S. Ma, Z. Chen, H. Sun, and N. K. Dutta, "High speed all optical logic gates based on quantum dot semiconductor optical amplifiers," Optics Express, vol. 18, no. 7, pp. 6417-6422, 2010.

[17] D. Nesset, M. C. Tatham, and D. Cotter, "All-optical AND gate operating on $10 \mathrm{Gbit} / \mathrm{s}$ signals at the same wavelength using four-wave mixing in a semiconductor laser amplifier," IEEE Electronics Letters, vol. 31, no. 11, pp. 896-897, 1995.

[18] H. Soto, C. A. Díaz, J. Topomondzo, D. Erasme, L. Schares, and G. Guekos, "All-optical AND gate implementation using crosspolarization modulation in a semiconductor optical amplifier," IEEE Photonics Technology Letters, vol. 14, no. 4, pp. 498-500, 2002.

[19] X. Zhang, Y. Wang, J. Sun, D. Liu, and D. Huang, "All-optical AND gate at $10 \mathrm{Gbit} / \mathrm{s}$ based on cascaded single-port-coupled SOAs," Optics Express, vol. 12, no. 3, pp. 361-366, 2004.

[20] L. Q. Guo and M. J. Connelly, "All-optical and gate with improved extinction ratio using signal induced nonlinearities in a bulk semiconductor optical amplifier," Optics Express, vol. 14, no. 7, pp. 2938-2943, 2006.

[21] J. H. Kim, Y. I. Kim, Y. T. Byun et al., "All-optical logic gates using semiconductor optical-amplifier-based devices and their applications," Journal of the Korean Physical Society, vol. 45, no. 5 I, pp. 1158-1161, 2004.

[22] X. Chen, L. Huo, Z. Zhao, L. Zhuang, and C. Lou, "Study on $100-\mathrm{Gb} / \mathrm{s}$ reconfigurable all-optical logic gates using a single semiconductor optical amplifier," Optics Express, vol. 24, no. 26, pp. 30245-30253, 2016.

[23] N. K. Dutta and Q. Wang, Semiconductor Optical Amplifiers, World Scientific Publishing Company, Singapore, 2nd edition, 2013.

[24] J. H. Kim, B. C. Kim, Y. T. Byun et al., "All-optical AND gate using cross-gain modulation in semiconductor optical amplifiers," Japanese Journal of Applied Physics, vol. 43, no. 2, pp. 608610, 2004.

[25] B.-K. Kang, J. H. Kim, Y. T. Byun et al., "All-optical AND gate using probe and pump signals as the multiple binary points in cross phase modulation," Japanese Journal of Applied Physics, vol. 41, no. 5 B, pp. L568-L570, 2002.

[26] E. Dimitriadou and K. E. Zoiros, "On the feasibility of 320 $\mathrm{Gb} / \mathrm{s}$ all-optical and gate using quantum-dot semiconductor optical amplifier-based mach-zehnder interferometer," Progress in Electromagnetics Research B, no. 50, pp. 113-140, 2013.

[27] J. Xu, X. Zhang, Y. Zhang, J. Dong, D. Liu, and D. Huang, "Reconfigurable all-optical logic gates for multi-input differential phase-shift keying signals: design and experiments," Journal of Lightwave Technology, vol. 27, no. 23, pp. 5268-5275, 2009.

[28] Z. Li, Y. Dong, C. Lu et al., "Comparison of cross-gain modulation effect of Manchester-duobinary, RZ-DPSK, NRZ-DPSK, RZ, and NRZ modulation formats in SOAs," IEEE Photonics Technology Letters, vol. 18, no. 24, pp. 2680-2682, 2006.

[29] D. Kong, Y. Li, H. Wang et al., "All-optical XOR gates for QPSK signal based optical networks," IEEE Electronics Letters, vol. 49, no. 7, pp. 485-486, 2013.

[30] X. Chen, Y. Yu, and X. Zhang, "All-optical logic minterms for three-input demodulated differential phase-shift keying signals at $40 \mathrm{~Gb} / \mathrm{s}$," IEEE Photonics Technology Letters, vol. 23, no. 2, pp. 118-120, 2011.

[31] I. Kang, C. Dorrer, and J. Leuthold, "All-optical XOR operation of $40 \mathrm{Gbit} / \mathrm{s}$ phase-shift-keyed data using four-wave mixing in semiconductor optical amplifier," IEEE Electronics Letters, vol. 40, no. 8, pp. 496-498, 2004.

[32] K. Chan, C.-K. Chan, L. K. Chen, and F. Tong, "Demonstration of $20-\mathrm{Gb} / \mathrm{s}$ all-optical XOR gate by four-wave mixing in semiconductor optical amplifier with RZ-DPSK modulated inputs," IEEE Photonics Technology Letters, vol. 16, no. 3, pp. 897-899, 2004.

[33] N. Deng, K. Chan, C.-K. Chan, and L.-K. Chen, "An all-optical XOR logic gate for high-speed RZ-DPSK signals by FWM in semiconductor optical amplifier," IEEE Journal of Selected Topics in Quantum Electronics, vol. 12, no. 4, pp. 702-707, 2006.

[34] A. Kotb, "An OR gate for $100 \mathrm{~Gb} / \mathrm{s}$ phase-shift keying signals in semiconductor optical amplifier-based delayed interferometer," Australian Basic and Applied Science, vol. 8, pp. 449-453, 2014.

[35] A. Kotb, "Analysis of all-optical logic XOR gate for $100 \mathrm{~Gb} / \mathrm{s}$ phase-shift keying modulated data signals in semiconductor optical amplifier-based Mach-Zehnder interferometer," Optical and Quantum Electronics, vol. 47, no. 5, pp. 1063-1070, 2015.

[36] W. Li, H. Hu, X. Zhang, and N. K. Dutta, "High speed all optical logic gates using binary phase shift keyed signal based on QD-SOA, International Journal of High Speed Electronics and Systems, vol. 24, no. 3-4, Article ID 1550005, 2015.

[37] G. Talli and M. J. Adams, "Amplified spontaneous emission in semiconductor optical amplifiers: modelling and experiments," Optics Communications, vol. 218, no. 1-3, pp. 161-166, 2003.

[38] E. Dimitriadou and K. E. Zoiros, "On the design of reconfigurable ultrafast all-optical NOR and NAND gates using a single 
quantum-dot semiconductor optical amplifier-based MachZehnder interferometer," Journal of Optics (United Kingdom), vol. 14, no. 10, Article ID 105401, 2012.

[39] T. Houbavlis, K. E. Zoiros, G. Kanellos, and C. Tsekrekos, "Performance analysis of ultrafast all-optical Boolean XOR gate using semiconductor optical amplifier-based Mach-Zehnder Interferometer," Optics Communications, vol. 232, no. 1-6, pp. 179-199, 2004.

[40] R. Bonk, T. Vallaitis, J. Guetlein et al., "The input power dynamic range of a semiconductor optical amplifier and its relevance for access network applications," IEEE Photonics Journal, vol. 3, no. 6, pp. 1039-1053, 2011. 

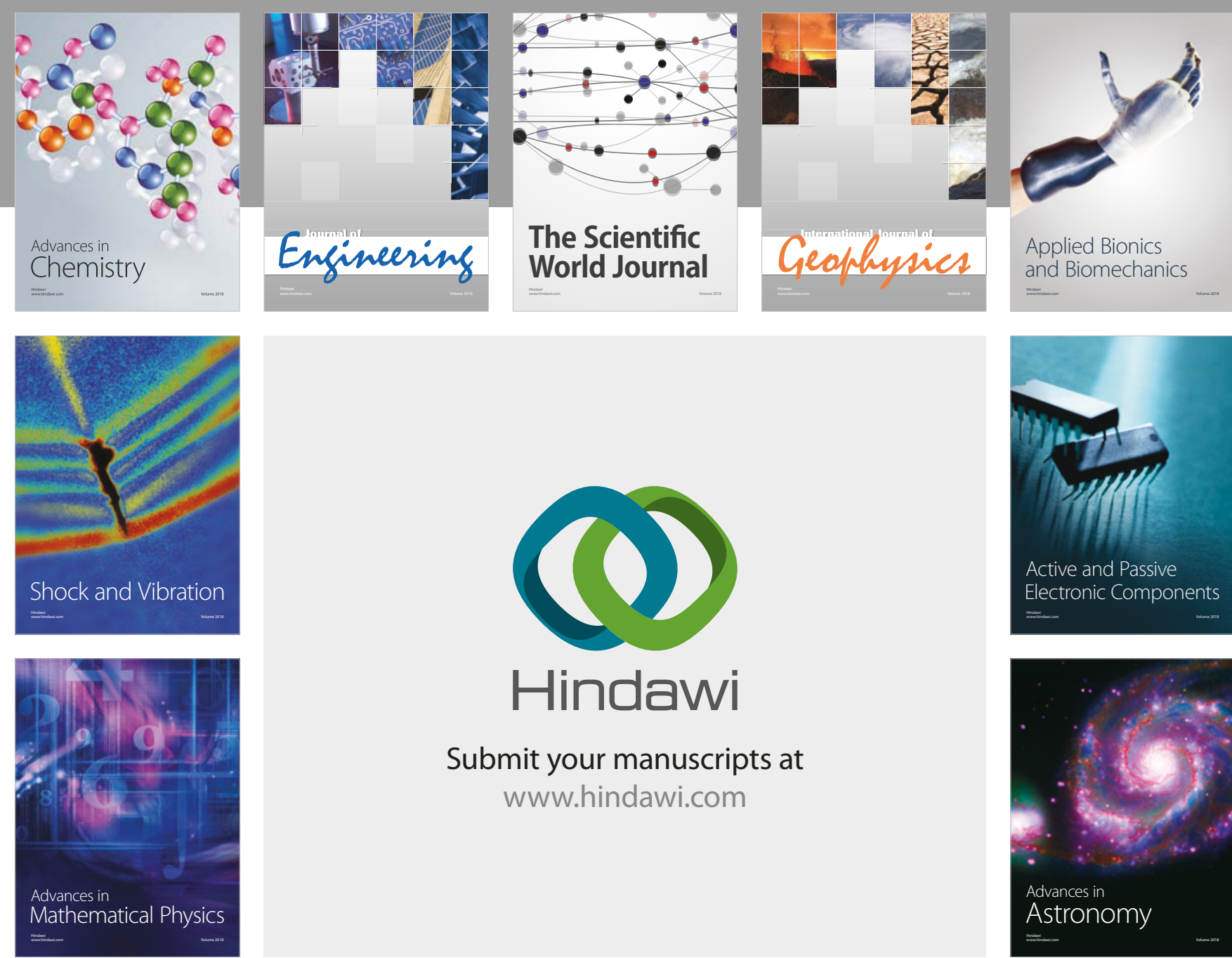

Submit your manuscripts at

www.hindawi.com

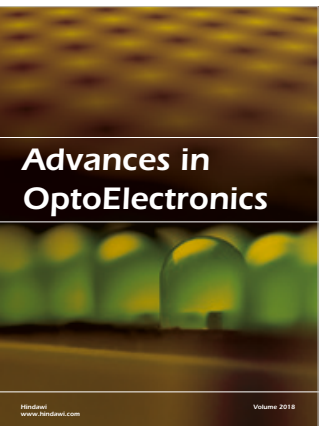

\section{Rotcting Machinery}
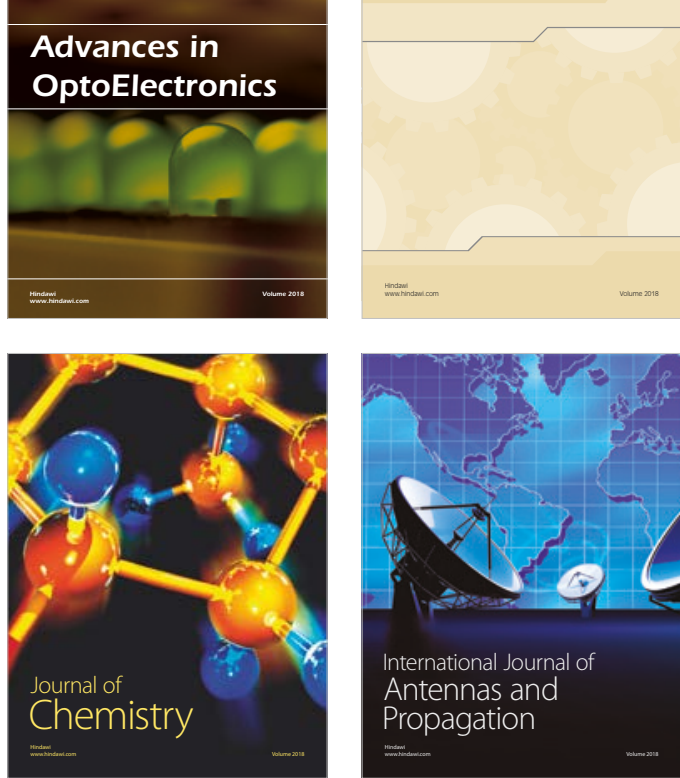

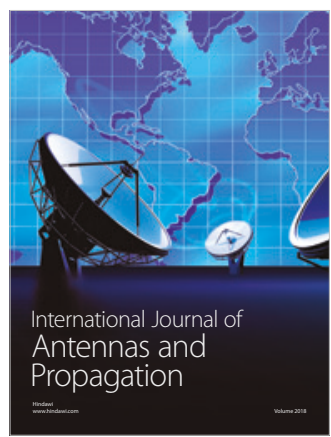

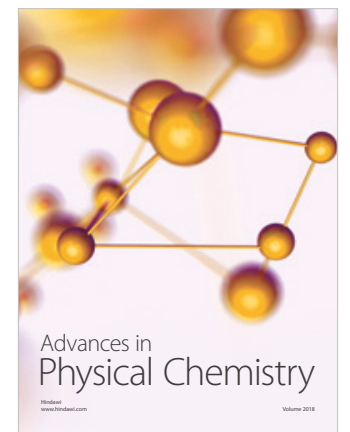

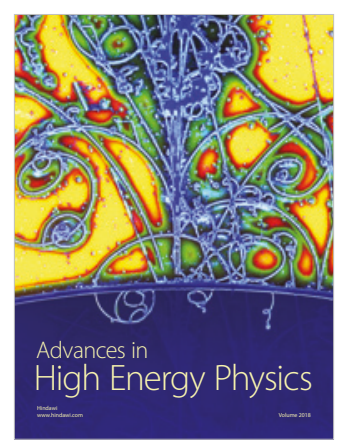

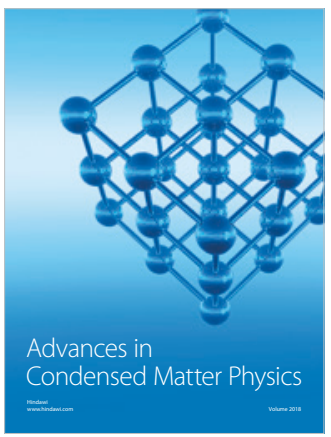

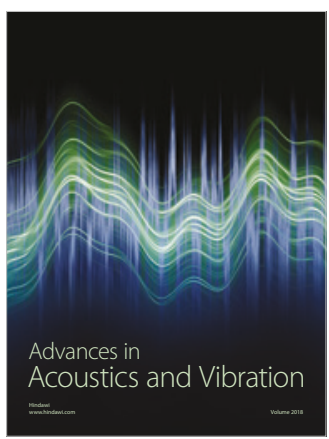

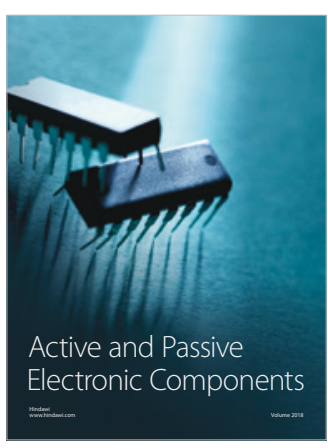
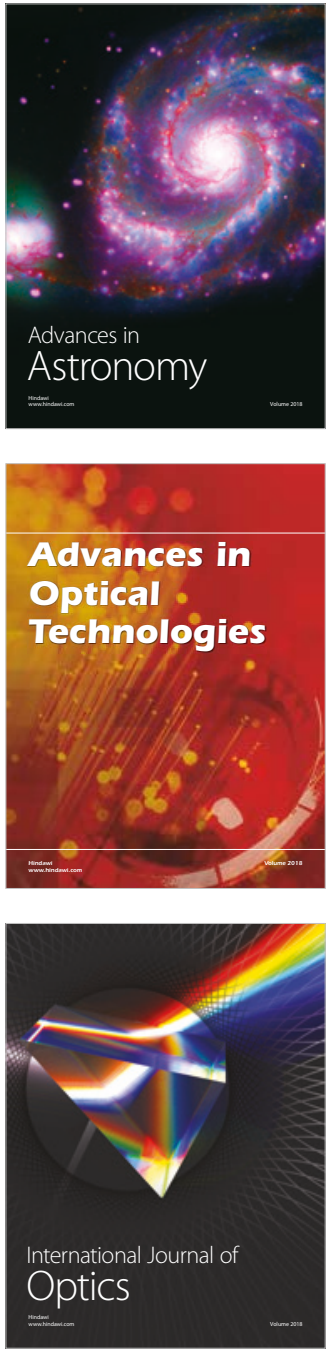